\title{
ON A COMPARISON THEOREM FOR SELF-ADJOINT PARTIAL DIFFERENTIAL EQUATIONS OF ELLIPTIC TYPE
}

\section{PHILIP HARTMAN AND AUREL WINTNER}

Let $T$ be a bounded, open set in the real, $n$-dimensional $x$-space, where $x=\left(x^{1}, \cdots, x^{n}\right)$, and suppose that the boundary $S$ of $T$ has a continuous normal. Let there be given on $T+S$ a continuous scalar $f(x)$ and a positive-definite line-element

$$
g_{\alpha \beta}(x) d x^{\alpha} d x^{\beta}
$$

with continuous coefficients $g_{i k}(x)=g_{k i}(x)$. The general form of a linear, elliptic, self-adjoint partial differential equation of second order on $T$ is

$$
\left(g^{\alpha \beta} u_{\alpha}\right)_{\beta}+f u=0,
$$

where $\left(g^{i k}\right)=\left(g_{i k}\right)^{-1}$ and ${ }_{\gamma}=\partial / \partial x^{\gamma}$.

Since the functions $g_{i k}(x)$ need not be differentiable, (2) cannot in general be written in the form

$$
g^{\alpha \beta} u_{\alpha \beta}+g^{\alpha \beta} u_{\alpha}+f u=0 .
$$

Correspondingly, it is not natural to speak of a solution $u(x)$ of (2) only if its second derivatives, which occur in (3) but not in (2), exist. A "solution" $u(x)$ of (2) will therefore be meant in the following sense: grad $u(x)$ is uniformly continuous on $T$ (hence can be extended, by continuity, to $T+S$ ), and the $n^{2}$ partial derivatives

$$
\left(g^{\alpha \beta}(x) u_{\alpha}(x)\right)_{\beta}
$$

exist, are continuous, and satisfy (2), at every point $x$ of $T$ (they need not exist on $S$ ).

Consider now, besides (2), another differential equation of the same form, say

$$
\left(G^{\alpha \beta} U_{\alpha}\right)_{\beta}+F U=0,
$$

where $\left(G_{i k}(x)\right)$ and $F(x)$ satisfy on $T+S$ the same conditions as $\left(g_{i k}(x)\right)$ and $f(x)$. Suppose that, on $T+S$,

$$
f \leqq F
$$

and

Received by the editors January 19, 1955. 


$$
\left(g_{i k}\right) \leqq\left(G_{i k}\right),
$$

where (6) means that the matrix $\left(G_{i k}-g_{i k}\right)$ is non-negative definite. Suppose that either the equality sign of (5) does not hold for some $x$ or, if it holds for all $x$, that is, if $f \equiv F$, then there exists some $x=x_{0}$ at which the sign of equality does not hold in (6) (so that $\left(G_{i k}-g_{i k}\right)$ is positive definite at $x_{0}$ ) and that, at the same $x_{0}$, the (common) value of $F$ (and $f$ ) does not vanish. If these conditions are satisfied, then (4) will be called $a$ strict Sturmian majorant of (2). This nomenclature is justified by the fact that, if $n=1$ in $x=\left(x^{1}, \cdots, x^{n}\right)$, then the conditions specified reduce to the assumptions under which the strict form of Sturm's classical comparison theorem is true for the pair of ordinary differential equations to which the partial differential equations (2), (4) reduce when $n=1$.

It will be shown that if (4) is a strict Sturmian majorant of (2) on $T$ and if there exists $a u(x)$ which does not vanish identically on any open set contained in $T$ and satisfies the boundary condition $u=0$ on $S$, then every $U(x)$ must vanish somewhere on $T+S$. Clearly, this theorem reduces to Sturm's classical result in the limiting case, $n=1$, of ordinary differential equations; cf. [1, p. 419].

REMARK. The wording of this theorem could be made simpler and sharper under the following assumption: Every solution of (2) which is 0 on some open subset of the (connected) open set $T$ is 0 on $T$. This assumption is known to be satisfied if $n=2$ and, in addition, the coefficients of (2) satisfy certain assumptions of smoothness. We do not know, however, either of a positive result or of a counterexample in the general case.

The proof of the theorem will depend on an elaboration of a device used by Picard $[2$, p. 151] in a similar context.

In order to prove the theorem, suppose that its assertion is false. Then there exists some $U$ which does not vanish on $T+S$. Hence the Green identity, which leads from the boundary condition $\left.u\right|_{s}=0$ to the divergence relation

$$
\int_{T}\left(w h^{\alpha}\right)_{\alpha} d T=0, \quad \text { where } w=u^{2},
$$

is applicable to the vector function $\left(h^{1}, \cdots, h^{n}\right)$ defined by

$$
h^{i}=-G^{i \beta} U_{\beta} / U \text {. }
$$

(Note that $U_{\beta} / U=(\log U)_{\beta}$ and that, correspondingly, (15) below then reduces to Riccati's differential equation.)

Since $u$ is a solution of (2) and.vanishes on $S$, another application of Green's identity shows that 


$$
\int_{T}\left(g^{\alpha \beta} u_{\alpha} \iota_{\beta}-f u^{2}\right) d T=0 .
$$

At every point $x=\left(x^{1}, \cdots, x^{n}\right)$ of $T$, let $\phi=\phi\left(v^{1}, \cdots, v^{n}, v^{n+1} ; x\right)$ be the $(n+1)$-ary quadratic form having the matrix

$$
\left(\begin{array}{cc}
\left(g^{i k}\right) & h^{\prime} \\
h & j
\end{array}\right),
$$

where $h^{\prime}$ denotes the transpose of the row vector $h=\left(h^{1}, \cdots, h^{n}\right)$ and $j$ is the scalar

$$
j=\operatorname{div} h-f, \quad\left(\operatorname{div} h=h^{\alpha}{ }_{\alpha}\right) .
$$

Then it is clear from (7) and (9) that

$$
\int_{T} \phi\left(u_{1}(x), \cdots, u_{n}(x), u(x) ; x\right) d T=0 .
$$

The theorem will now be proved by showing that its assumptions contradict (12).

First, if $x$ is fixed, then, since $\left(g^{i k}\right)$ is positive definite, it is clear that (10) is positive definite or positive semi-definite according as the determinant of $(10)$ is positive or zero. If the $i$ th row, $\left(g^{i 1}, \cdots, g^{i n}, h^{i}\right)$, of (10) is multiplied by $g_{i \alpha} h^{\alpha}$ and subtracted from the $(n+1)$ st row, $\left(h^{1}, \cdots, h^{n}, j\right)$, for $i=1, \cdots, n$, then the last row is changed to $\left(0, \cdots, 0, j-g_{i \alpha} h^{\alpha} h^{i}\right)$, since $g_{i \alpha} h^{\alpha} g^{i k}=h^{i}$. Hence the determinant of (10) can be factored into

$$
\left(\operatorname{det} g^{i k}\right)\left(j-g_{\alpha \beta} h^{\alpha} h^{\beta}\right) \text {. }
$$

Since the first factor in (13) is positive, insertion of (11) into the second factor of (13) shows that (10) is positive definite or semidefinite (at a given $x$ ) according as

$$
-h_{\alpha}^{\alpha}+g_{\alpha \beta} h^{\alpha} h^{\beta}+f \leqq 0 .
$$

Next, it is readily verified from (8) that (4) can be written in the form

$$
-h_{\alpha}{ }^{\alpha}+G_{\alpha \beta} h^{\alpha} h^{\beta}+F=0 .
$$

If (14) is subtracted from (15), it follows that, at a given point $x$ of $T$, the quadratic form $\phi\left(v^{1}, \cdots, v^{n}, v^{n+1} ; x\right)$ is positive definite or semidefinite according as, at that point $x$ of $T$,

$$
\left(G_{\alpha \beta}-g_{\alpha \beta}\right) h^{\alpha} h^{\beta}+(F-f) \geqq 0 .
$$

In view of (5) and (6), both terms on the left of (16) are non- 
negative. Hence (16) is satisfied throughout $T$. This means that the quadratic form $\phi$ is non-negative definite throughout. It follows therefore from (12) that

$$
\phi\left(u_{1}(x), \cdots, u_{n}(x), u(x) ; x\right)=0
$$

holds at every point $x$ of $T$. That this contains a contradiction will now be shown by using the alternative proviso specified after (5) $-(6)$.

The first case of that alternative is that $f<F$ holds at some point $x_{0}$ of $T$, hence at every point of some neighborhood $R$ of $x_{0}$. Thus, at every point $x$ of $R$, the sign of equality fails to hold in (16). Hence $\phi\left(v^{1}, \cdots, v^{n}, v^{n+1} ; x\right)$ is positive definite at every point $x$ of $R$. In view of (17), this implies that $u(x) \equiv 0$ on $R$. This contradicts, however, one of the assumptions of the italicized theorem.

The second case of the alternative is the following situation: $f=F$ holds on $T$ but $\left(g_{i k}\right)<\left(G_{i k}\right)$ and $F \neq 0$ on $R$. Then, if (16) and (17) are used as before, there results (even if $F \neq 0$ is not used as yet) the same contradiction as in the first case, except when all $n$ functions $h^{i}=h^{i}(x)$, occurring in the positive definite form which is the first term in (16), vanish on $R$ identically. But this possibility cannot take place. For, if it did, it would follow from (8) that every $U_{i}=U_{i}(x)$, that is, grad $U(x)$, must vanish on $R$ identically; so that $U(x)=$ const. on $R$. On the other hand, $U \neq 0$ on $T$, by an assumption of the italicized theorem. Hence $U$ is a nonvanishing constant on $R$. In view of (4), this implies that $F \equiv 0$ on $R$. Since this contradicts the assumption, the proof is complete.

\section{REFERENCES}

1. E. Kamke, $A$ new proof of Sturm's comparison theorems, Amer. Math. Monthly vol. 46 (1939) pp. 417-421.

2. E. Picard, Memoire sur la theorie des equations aux derives partielles et la methode des approximations successives, J. Math. Pures Appl. vol. 6 (1890) pp. 145-210

The Johns Hopkins University 